\title{
EDITORIAL
}

\section{Moving from one to many: insights from the growing list of pleiotropic cancer risk genes}

Pleiotropy, a phenomenon in which a single gene affects multiple phenotypes, is becoming very common among different cancer types and cancer-related phenotypes, such as those in hormonal, cardiometabolic and inflammatory/immune conditions. The discovery of pleiotropic associations can improve our understanding of cancer and help to target investigation of genes with greater clinical relevance.

British Journal of Cancer (2019) 120:1087-1089; https://doi.org/10.1038/s41416-019-0475-9

\section{MAIN}

Closely following heart disease, cancer is the second leading cause of death in westernised populations. The complex biology of cancer is underscored by the discovery of more than 1000 lowpenetrance cancer risk variants. ${ }^{1}$ Estimates of shared genetic heritability between different cancer types have shown statistically significant correlations, with estimates as high as $r_{\mathrm{g}}=0.55$ for pancreatic and colorectal cancer. ${ }^{2}$ These estimates are consistent with expectations of extensive pleiotropy among polygenic traits. ${ }^{3}$ Accordingly, it is not surprising that genome-wide association studies (GWAS) have highlighted many commonalities in genetic risk and overlap in key pathways across cancer types. Some of the most prominent pleiotropic genes include MYC, TERT and HNF1B, all of which are linked to a growing number of cancer types. Recent cross-cancer GWAS have identified seven new pleiotropic genes that were not previously discovered by single-trait analysis, further demonstrating that this approach can power new discoveries. ${ }^{4}$

Despite the fact that pleiotropy is pervasive throughout the human genome, investigations to characterise the shared genetic basis of common cancers and other cancer-related phenotypes remain limited, but the plethora of pleiotropy findings revealed through ad hoc analyses (Fig. 1) suggest that many additional shared genetic risk genes exist. Here we highlight key examples of the insights gained from comprehensive and systematic crosscancer GWAS analyses. Pleiotropic discoveries can (1) identify shared biologic pathways and prioritise probable causal relationships, (2) reveal unexpected links between phenotypes and aid in aetiological disease classification, (3) test key assumptions for Mendelian randomisation studies, (4) inform repurposing of drugs and predict adverse drug reactions, and (5) increase the statistical power.

\section{SHARED BIOLOGICAL PATHWAYS AND UNEXPECTED PHENOTYPIC LINKS}

Pleiotropy has for long been described in monogenic diseases because high-penetrance mutations often cause a constellation of seemingly unrelated clinical features. ${ }^{5}$ As an example, PTEN hamartoma tumour syndrome (PHTS), which is caused by mutations in PTEN, predisposes to multiple cancers. PHTS is characterised by multiple hamartomas - benign tumour-like malformations comprising an abnormal mixture of cells and tissues - that can arise in any organ. Although PTEN is a tumour suppressor, it is also involved in non-canonical pathways, meaning that individuals with PHTS can also suffer from severe disfigurement and intellectual disability. ${ }^{6}$ This is referred to as biological pleiotropy (e.g. cancer $\leftarrow \mathrm{G}_{\text {PTEN }} \rightarrow$ intellectual disability). By contrast, pleiotropic associations can also arise when one phenotype influences another. Take, for instance, CHRNA5, a gene that associates with lung cancer, chronic obstructive pulmonary disease (COPD) and smoking behaviours. Associations with lung cancer could be due to the profound effects of CHRNA5 variants on smoking intensity, either directly or indirectly through effects on COPD, in a phenomenon referred to as mediated pleiotropy $\left(\mathrm{G}_{C H R N A} \rightarrow\right.$ smoking $\rightarrow$ COPD $\rightarrow$ lung cancer). Systematic analysis of possible pathways between $\mathrm{G}_{\text {CHRNA }}$ and lung cancer risk suggests that both direct and mediated effects contribute, with approximately $40 \%$ attributed to smoking (directly or through COPD). ${ }^{7}$ Systematic investigations can provide critical new insight into shared disease mechanisms, causal relationships or novel biological pathways. However, little attention has been given to the study of pleiotropy in complex phenotypes, as opposed to in Mendelian disease. GWAS have provided ample evidence that complex traits are highly polygenic, which has led to the establishment of very large case-control studies and encouraged super-consortia usually focusing on a single disease. The rapid discovery of variant associations by these 'disease-specific' consortia has, however, detracted from efforts to find pleiotropic key regulator genes with far-reaching aetiological influences, and hindered the ability to readily perform cross-trait analyses.

GWAS have identified many genetic risk factors that are shared between cancers and other related phenotypes, such as cardiometabolic (CDKN2B-AS1, HNF1B), inflammatory/immune (CDKN1B, FADS1), obesity (FTO), or hormonal (LGR5) conditions. Some of these associations initially seemed rather surprising, such as the positive link between prostate cancer and HNF1B, which also showed a reduced risk for type 2 diabetes; however, this result is consistent with the observation that individuals with type 2 diabetes are at decreased risk for prostate cancer $^{8}$ - an unexpected association that had previously been given limited attention.

\section{MENDELIAN RANDOMISATION}

The number of publications involving Mendelian randomisation studies has rapidly increased as of late; most likely, this 


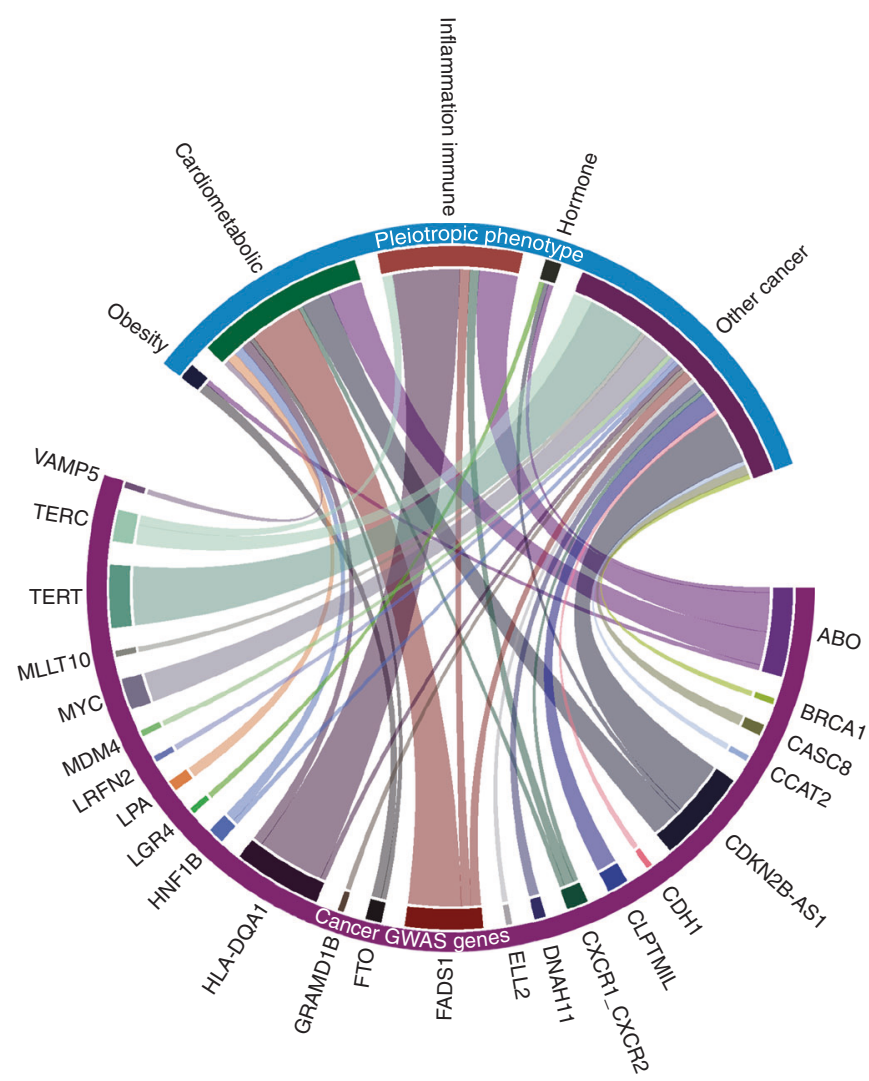

Fig. 1 Examples of pleiotropic genes near a GWAS-identified cancer locus associated with another cancer and/or phenotype in a relevant condition. Each gene represents a pleiotropic locus that is associated with multiple cancer types and/or one cancer type, and cancer-related traits and biomarkers. Connections in this chord diagram indicate that variants in or near respective genes associate with both a cancer type and one or more phenotypes within the linked conditions. The width of the chord corresponds to the number of phenotypes within the respective area; for example, the widest chord between TERT and Other cancer represents association between the TERT locus and 12 different cancers

reflects their purported ability to estimate causal effects in observational settings. In this capacity, Mendelian randomisation has been proposed as a pharmacovigilance and drugrepurposing tool to help identify treatment targets and to prioritise (or deprioritise) major investments in randomised controlled trials (RCTs). In this setting, Mendelian randomisation involves finding genetic variants associated with a modifiable target (e.g. plasma selenium and dietary supplementation), and then testing the association between those variants and the outcome (e.g. prostate cancer). ${ }^{9}$ However, the absence of pleiotropy is a core assumption that underlies Mendelian randomisation studies, and violation of this assumption can cause severe bias. For example, if the genetic variants used as a proxy for an intended target are associated with decreasing cancer risk through an alternative pathway, the drug or supplement in question could be completely ineffective, or even harmful, despite support from Mendelian randomisation. The extent of pleiotropy among complex traits and diseases is only beginning to be appreciated. As we typically only assess pleiotropy in the context of variants that have already been reported, more comprehensive cross-trait studies are needed before we continue to replace true RCTs with an imperfect statistical approach.

\section{DRUG REPURPOSING}

It is estimated that the success rate for drug development could be doubled if the selection of drug targets is supported by evidence from human genetic studies. ${ }^{10}$ The examples above demonstrate how the discovery of pleiotropic associations can improve RCT design, by screening for subtypes and adverse drug reactions. Identifying pleiotropy can also help to repurpose existing drugs, avoiding de novo development and further predict adverse drug events, thereby redirecting the efforts to more promising targets before the inception of an RCT.

The extent of pleiotropy between cancer loci and other seemingly disparate diseases and traits presented in Fig. 1 are intriguing. So far, few studies have performed genome-wide pleiotropic analyses between cancer traits and other complex diseases. Thus, because the results in Fig. 1 come from the comparison of results from single-trait GWAS, it is likely that the extent of pleiotropy is vastly underestimated given that pleiotropic analyses increase the statistical power for new discoveries. Therefore, the new era of GWAS should move away from the narrowly focused cataloguing of genotype and singlephenotype associations, and take into account comprehensive cross-trait analyses if we wish to fully realise the goals of precision medicine.

\section{AUTHOR CONTRIBUTIONS}

S.A.B drafting of manuscript. U.P critical revision of manuscript

\section{ADDITIONAL INFORMATION}

Competing interests: The authors declare no competing interests.

Ethics approval and consent to participate: Not applicable.

Data availability: Not applicable.

Funding: S.A.B and U.P. are funded by GECCO: National Cancer Institute, National Institutes of Health, U.S. Department of Health and Human Services (R01 CA201407).

Publisher's note: Springer Nature remains neutral with regard to jurisdictional claims in published maps and institutional affiliations.

Stephanie A. Bien ${ }^{1}$ and Ulrike Peters ${ }^{1,2}$

${ }^{1}$ Division of Public Health Sciences, Fred Hutchinson Cancer Research Center, Seattle, WA 98109, USA and ${ }^{2}$ Department of Epidemiology, School of Public Health, University of Washington, Seattle, WA 98109, USA

Correspondence: Stephanie A. Bien (sbien@fredhutch.org)

\section{REFERENCES}

1. MacArthur, J., Bowler, E., Cerezo, M., Gil, L., Hall, P., Hastings, E. et al. The new NHGRI-EBI Catalog of published genome-wide association studies (GWAS Catalog). Nucleic Acids Res. 45, D896-D901 (2017).

2. Lindström, S., Finucane, H., Bulik-Sullivan, B., Schumacher, F. R., Amos, C. I., Hung, R. J. et al. Quantifying the genetic correlation between multiple cancer types. Cancer Epidemiol. Biomark. Prev. 26, 1427-1435 (2017).

3. Barton, N. H. Pleiotropic models of quantitative variation. Genetics $124,773-782$ (1990).

4. Kar, S. P., Beesley, J., Amin Al Olama, A., Michailidou, K., Tyrer, J., Kote-Jarai, Zs et al. Genome-wide meta-analyses of breast, ovarian, and prostate cancer association studies identify multiple new susceptibility loci shared by at least two cancer types. Cancer Discov. 6, 1052-1067 (2016).

5. Visscher, P. M. \& Yang, J. A plethora of pleiotropy across complex traits. Nat. Genet. 48, 707-708 (2016). 
6. Hobert, J. A. \& Eng, C. PTEN hamartoma tumor syndrome: an overview. Genet. Med. 11, 687-694 (2009).

7. Wang, J., Spitz, M. R., Amos, C. I., Wu, X., Wetter, D. W., Cinciripini, P. M. et al. Method for evaluating multiple mediators: mediating effects of smoking and COPD on the association between the CHRNA5-A3 variant and lung cancer risk. PLOS ONE 7, e47705 (2012).

8. Pierce, B. L. \& Ahsan, H. Genetic susceptibility to type 2 diabetes is associated with reduced prostate cancer risk. Hum. Hered. 69, 193-201 (2010).

9. Yarmolinsky, J., Bonilla, C., Haycock, P. C., Langdon, R. J. Q., Lotta, L. A., Langenberg, $C$. et al. Circulating selenium and prostate cancer risk: A mendelian randomization analysis. J. Natl. Cancer Inst. 110, 1035-1038 (2018).

10. Nelson, M. R., Tipney, H., Painter, J. L., Shen, J., Nicoletti, P., Shen, Y. et al. The support of human genetic evidence for approved drug indications. Nat. Genet. 47, 856-860 (2015).
Open Access This article is licensed under a Creative Commons Attribution 4.0 International License, which permits use, sharing, adaptation, distribution and reproduction in any medium or format, as long as you give appropriate credit to the original author(s) and the source, provide a link to the Creative Commons license, and indicate if changes were made. The images or other third party material in this article are included in the article's Creative Commons license, unless indicated otherwise in a credit line to the material. If material is not included in the article's Creative Commons license and your intended use is not permitted by statutory regulation or exceeds the permitted use, you will need to obtain permission directly from the copyright holder. To view a copy of this license, visit http://creativecommons. org/licenses/by/4.0/.

(c) The Author(s) 2019 